\title{
EXTENSION OF THE TARGET SCATTERING VECTOR MODEL TO THE BISTATIC CASE
}

\author{
Lionel Bombrun ${ }^{1,2}$ \\ ${ }^{1}$ Grenoble-Image-sPeech-Signal-Automatics Lab, CNRS \\ GIPSA-lab DIS/SIGMAPHY, Grenoble INP - BP 46, 38402 Saint-Martin-d'Hères, FRANCE \\ Tel: +33 476826424 - Fax: +33 476574790 - Email: lionel.bombrun@ gipsa-lab.grenoble-inp.fr \\ ${ }^{2}$ SONDRA Research Alliance \\ Plateau du Moulon, 3 rue Joliot-Curie, 91192 Gif-sur-Yvette Cedex, FRANCE \\ Tel: +33169851804 - Fax: +33169851809
}

\begin{abstract}
The polarimetric information has been widely used to interpret the Synthetic Aperture Radar (SAR) scene. Hence, many decompositions have been introduced to extract polarimetric parameters with a physical meaning. Nevertheless, for most of them, the reciprocity assumption is assumed. For a bistatic PolSAR sensor, the cross-polarization terms of the scattering matrix are not equal. This paper presents a generalization of the Target Scattering Vector Model (TSVM) to the bistatic case.
\end{abstract}

Index Terms - Bistatic Polarimetry, Polarimetric Synthetic Aperture Radar, Roll-invariant decomposition, Target Scattering Vector Model.

\section{INTRODUCTION}

In the context of Polarimetric Synthetic Aperture Radar (PolSAR) imagery, the extraction of roll-invariant parameters is one of the major point of interest for segmentation, classification and detection. In 2007, for the monostatic case, Ridha Touzi has proposed a new Target Scattering Vector Model (TSVM) [1]. Based on the Kennaugh-Huynen decomposition, this model allows to extract four roll-invariant parameters.

For the bistatic case, the reciprocity assumption is in general no more valid. This paper presents a extension of the TSVM when the cross-polarization terms are not equal. First, a presentation of bistatic polarimetry is exposed by means of the Kennaugh-Huynen decomposition [2]. Then, the TSVM is introduced as a projection of the scattering matrix in the Pauli basis to extract roll-invariant parameters [1] and a comparison with the monostatic case is carried out. Next, a presentation of the computation of the TSVM parameters is exposed. Finally, some comparisons with the classical $\alpha / \beta$ model are shown.

\section{THE KENNAUGH-HUYNEN CON-DIAGONALIZATION}

Coherent targets are fully described by their scattering matrix $\mathbf{S}$. In the context bistatic polarimetry, $\mathbf{S}$ is a complex $2 \times 2$ matrix, $\mathbf{S}=\left[\begin{array}{ll}S_{H H} & S_{H V} \\ S_{V H} & S_{V V}\end{array}\right]$ where the cross-polarization elements $S_{H V}$ and $S_{V H}$ are not equal in general.

Kennaugh and Huynen have proposed to apply the characteristic decomposition on the scattering matrix to retrieve physical parameters [2] [3] [4]. The Kennaugh-Huynen decomposition is parametrized by means of 8 independent parameters: $\theta_{R}, \tau_{R}, \theta_{E}, \tau_{E}, \nu, \mu, \kappa$ and $\gamma$ by [2] [5] [6]:

$$
\mathbf{S}=e^{-j \theta_{R} \sigma_{3}} e^{-j \tau_{R} \sigma_{2}} e^{-j \nu \sigma_{1}} \mathbf{S}_{\mathbf{0}} e^{j \nu \sigma_{1}} e^{-j \tau_{E} \sigma_{2}} e^{j \theta_{E} \sigma_{3}}
$$

where:

$\mathbf{S}_{\mathbf{0}}=\mu e^{j \kappa}\left[\begin{array}{cc}1 & 0 \\ 0 & \tan ^{2} \gamma\end{array}\right]$ and $e^{j \alpha \sigma_{k}}=\sigma_{0} \cos \alpha+j \sigma_{k} \sin \alpha$.

$\sigma_{i}$ are the spin Pauli matrices defined by:

$$
\begin{aligned}
& \sigma_{1}=\left[\begin{array}{ll}
1 & 0 \\
0 & 1
\end{array}\right], \sigma_{2}=\left[\begin{array}{cc}
1 & 0 \\
0 & -1
\end{array}\right], \\
& \sigma_{3}=\left[\begin{array}{ll}
0 & 1 \\
1 & 0
\end{array}\right], \sigma_{4}=\left[\begin{array}{cc}
0 & -j \\
j & 0
\end{array}\right] .
\end{aligned}
$$

$\theta_{R}$ and $\theta_{E}$ are the tilt angles. $\tau_{R}$ and $\tau_{E}$ are the helicity. The subscript $R$ and $E$ stand respectively for reception and emission. $\mu$ is the maximum amplitude return. $\gamma$ and $\nu$ are respectively referred as the characteristic and skip angles. $\kappa$ is the absolute phase of the target, this term is generally ignored except for interferometric applications.

Moreover, it can be shown that:

$$
\begin{aligned}
e^{-j \nu \sigma_{1}} \mathbf{S}_{0} e^{j \nu \sigma_{1}} & =\left[\begin{array}{cc}
\mu e^{2 j(\nu+\kappa / 2)} & 0 \\
0 & \mu \tan ^{2} \gamma e^{-2 j(\nu-\kappa / 2)}
\end{array}\right] \\
& =\left[\begin{array}{cc}
\lambda_{1} & 0 \\
0 & \lambda_{2}
\end{array}\right]
\end{aligned}
$$


where $\lambda_{1}$ and $\lambda_{2}$ are the two complex con-eigenvalues of $\mathbf{S}$.

\section{THE TARGET SCATTERING VECTOR MODEL}

\subsection{Definition}

The TSVM consists in the projection in the Pauli basis of the scattering matrix con-diagonalized by the Takagi method. It yields that $\mathbf{k}_{\mathbf{P}}=1 / \sqrt{2}\left[S_{H H}+S_{V V}, S_{H H}-S_{V V}, S_{H V}+\right.$ $\left.S_{V H}, j\left(S_{H V}-S_{V H}\right)\right]^{T}$. After some mathematical manipulations, one can express the target vector $\mathbf{k}_{\mathbf{P}}$ by means of Huynen's parameters (See (5) at the top of the next page).

By following the same procedure as proposed by Touzi in [1], one can introduce the symmetric scattering type magnitude and phase parameters, denoted respectively $\alpha_{s}$ and $\Phi_{\alpha_{s}}$ by:

$$
\tan \left(\alpha_{s}\right) e^{j \Phi_{\alpha_{s}}}=\frac{\lambda_{1}-\lambda_{2}}{\lambda_{1}+\lambda_{2}} .
$$

According to (5), one can decompose $\mathbf{k}_{\mathbf{P}}$ as the product of three terms (see (7) at the top of the next page). $\Phi_{s}$ corresponds to the phase of $\lambda_{1}+\lambda_{2}$. It can be noticed that the first and second terms are "rotation" matrices which depend only on the tilt angles $\theta_{R}$ and $\theta_{E}$.

\subsection{Roll-invariant target vector}

As a consequence, for the bistatic case, the expression of the roll-invariant target vector $\mathbf{k}_{\mathbf{P}}^{\text {roll-inv }}$ is given by:

$$
\mathbf{k}_{\mathbf{P}}^{\text {roll-inv }}=\mu\left[\begin{array}{c}
\cos \alpha_{s} \cos \left(\tau_{1}\right) \\
\sin \alpha_{s} e^{j \Phi_{\alpha_{s}}} \cos \left(\tau_{2}\right) \\
-j \cos \alpha_{s} \sin \left(\tau_{1}\right) \\
-j \sin \alpha_{s} e^{j \Phi_{\alpha_{s}}} \sin \left(\tau_{2}\right)
\end{array}\right],
$$

where $\tau_{1}=\tau_{R}+\tau_{E}$ and $\tau_{2}=\tau_{R}-\tau_{E}$. In the context of bistatic polarimetry, five parameters (namely $\mu, \tau_{R}, \tau_{E}, \alpha_{s}$ and $\Phi_{\alpha_{s}}$ ) are necessary for an unambiguous description of a coherent target.

\subsection{Link with the monostatic case}

The monostatic case can be retrieved from the bistatic case by assuming $\theta=\theta_{R}=\theta_{E}$ and $\tau_{m}=\tau_{R}=\tau_{E}$. Consequently, when the reciprocity assumption holds, the roll-invariant target vector, introduced by Touzi, is obtained:

$$
\mathbf{k}_{\mathbf{P}}^{\text {roll-inv }}=\mu\left[\begin{array}{c}
\cos \alpha_{s} \cos \left(2 \tau_{m}\right) \\
\sin \alpha_{s} e^{j \Phi_{\alpha_{s}}} \\
-j \cos \alpha_{s} \sin \left(2 \tau_{m}\right) \\
0
\end{array}\right] .
$$

\section{TSVM PARAMETERS COMPUTATION}

\subsection{The Kennaugh matrix}

The Kennaugh matrix $\mathbf{K}$ is another representation of the scattering matrix $\mathbf{S}$, its expression is given by $\mathbf{K}=2 \mathbf{A}^{*} \mathbf{W A}^{-1}$ with $\mathbf{W}=\mathbf{S} \otimes \mathbf{S}$. $\otimes$ is the Kronecker product, and:

$$
\mathbf{A}=\left[\begin{array}{cccc}
1 & 0 & 0 & 1 \\
1 & 0 & 0 & -1 \\
0 & 1 & 1 & 0 \\
0 & j & -j & 0
\end{array}\right]
$$

\subsection{The Kennaugh matrices of orders 0 to 2}

Let $\mathbf{O}_{1}, \mathbf{O}_{2}$ and $\mathbf{O}_{3}$ be the three "rotation matrices" defined by [5]:

$$
\begin{aligned}
& \mathbf{O}_{\mathbf{1}}(2 \nu)=\left[\begin{array}{cccc}
1 & 0 & 0 & 0 \\
0 & 1 & 0 & 0 \\
0 & 0 & \cos (2 \nu) & -\sin (2 \nu) \\
0 & 0 & \sin (2 \nu) & \cos (2 \nu)
\end{array}\right] \\
& \mathbf{O}_{\mathbf{2}}(2 \tau)=\left[\begin{array}{cccc}
1 & 0 & 0 & 0 \\
0 & \cos (2 \tau) & 0 & \sin (2 \tau) \\
0 & 0 & 1 & 0 \\
0 & -\sin (2 \tau) & 0 & \cos (2 \tau)
\end{array}\right] \\
& \mathbf{O}_{\mathbf{3}}(2 \theta)=\left[\begin{array}{cccc}
1 & 0 & 0 & 0 \\
0 & \cos (2 \theta) & -\sin (2 \theta) & 0 \\
0 & \sin (2 \theta) & \cos (2 \theta) & 0 \\
0 & 0 & 0 & 1
\end{array}\right] .
\end{aligned}
$$

The Kennaugh matrices of orders 0 to 2 , denoted $\mathbf{K}^{(\mathbf{i})}$, are defined by:

$$
\left\{\begin{array}{l}
\mathbf{K}^{(\mathbf{2})}=\mathbf{O}_{\mathbf{3}}\left(-2 \theta_{R}\right) \mathbf{K} \mathbf{O}_{\mathbf{3}}\left(2 \theta_{E}\right) \\
\mathbf{K}^{(\mathbf{1})}=\mathbf{O}_{\mathbf{2}}\left(2 \tau_{R}\right) \mathbf{K}^{(\mathbf{2})} \mathbf{O}_{\mathbf{2}}\left(-2 \tau_{E}\right) \\
\mathbf{K}^{(\mathbf{0})}=\mathbf{O}_{\mathbf{1}}(-2 \nu) \mathbf{K}^{(\mathbf{1})} \mathbf{O}_{\mathbf{1}}(2 \nu)
\end{array}\right.
$$

\subsection{TSVM parameters computation}

\subsubsection{Tilt angles}

In practice, thanks to the scattering scattering matrix $\mathbf{S}$, the Kennaugh matrix $\mathbf{K}$ is first computed. The tilt angles $\theta_{E}$ and $\theta_{R}$ are then directly deduced from the Kennaugh matrix $\mathbf{K}$ by [7]:

$$
\tan \left(2 \theta_{E}\right)=\frac{\mathbf{K}_{02}}{\mathbf{K}_{01}} \text { and } \tan \left(2 \theta_{R}\right)=\frac{\mathbf{K}_{20}}{\mathbf{K}_{10}} .
$$

In (15), $\mathbf{K}_{i j}$ corresponds to the element of $\mathbf{K}$ at position $(i+$ $1, j+1)$. Once $\theta_{E}$ and $\theta_{R}$ are found, the Kennaugh matrix of order 2, namely $\mathbf{K}^{(2)}$, is computed according to (14). As this matrix does not depend on the tilt angles, it can be viewed as the roll-invariant Kennaugh matrix. 


$$
\begin{aligned}
& \mathbf{k}_{\mathbf{P}}=\frac{1}{\sqrt{2}}\left[\begin{array}{c}
\left(\lambda_{1}+\lambda_{2}\right) \cos \left(\tau_{R}+\tau_{E}\right) \cos \left(\theta_{R}-\theta_{E}\right)+j\left(\lambda_{1}-\lambda_{2}\right) \sin \left(\tau_{E}-\tau_{R}\right) \sin \left(\theta_{E}-\theta_{R}\right) \\
\left(\lambda_{1}-\lambda_{2}\right) \cos \left(\tau_{R}-\tau_{E}\right) \cos \left(\theta_{R}+\theta_{E}\right)+j\left(\lambda_{1}+\lambda_{2}\right) \sin \left(\tau_{R}+\tau_{E}\right) \sin \left(\theta_{R}+\theta_{E}\right) \\
\left(\lambda_{1}-\lambda_{2}\right) \cos \left(\tau_{R}-\tau_{E}\right) \sin \left(\theta_{R}+\theta_{E}\right)-j\left(\lambda_{1}+\lambda_{2}\right) \sin \left(\tau_{R}+\tau_{E}\right) \cos \left(\theta_{R}+\theta_{E}\right) \\
\left(\lambda_{1}-\lambda_{2}\right) \sin \left(\tau_{E}-\tau_{R}\right) \cos \left(\theta_{R}-\theta_{E}\right)+j\left(\lambda_{1}+\lambda_{2}\right) \cos \left(\tau_{r}+\tau_{E}\right) \sin \left(\theta_{E}-\theta_{R}\right)
\end{array}\right]
\end{aligned}
$$

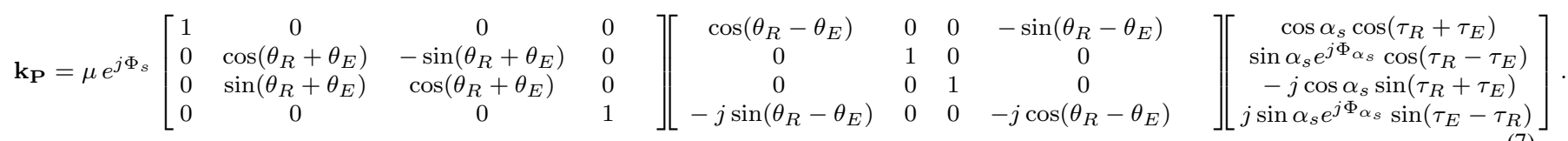

\subsubsection{Helicity angles}

Similarly, the helicity angles $\tau_{R}$ are $\tau_{E}$ are issued from the Kennaugh matrix of order 2 by [7]:

$$
\tan \left(2 \tau_{R}\right)=\frac{\mathbf{K}_{30}^{(2)}}{\mathbf{K}_{10}^{(2)}} \text { and } \tan \left(2 \tau_{E}\right)=\frac{\mathbf{K}_{03}^{(2)}}{\mathbf{K}_{01}^{(2)}} .
$$

\subsubsection{Characteristic and skip angles}

Next, the skip and characteristic angles $(\nu$ and $\gamma)$ are deduced from the Kennaugh matrices of order 1 and 0 by:

$$
\tan (4 \nu)=\frac{\mathbf{K}_{32}^{(1)}}{\mathbf{K}_{33}^{(1)}} \text { and } \cos (2 \gamma)=A \pm \sqrt{A^{2}-1}
$$

with $A=\frac{\mathbf{K}_{11}^{(0)}}{\mathbf{K}_{01}^{(0)}}$. The solution adopted is the $A \pm \sqrt{A^{2}-1}$ ranging in the interval $[-1,1]$.

\subsubsection{Symmetric scattering type magnitude and phase}

Finally, the symmetric scattering type magnitude and phase, $\alpha_{s}$ and $\Phi_{\alpha_{s}}$, are directly deduced from parameters $\nu$ and $\gamma$ by:

$$
\tan \left(\alpha_{s}\right) e^{j \Phi_{\alpha_{s}}}=\frac{\lambda_{1}-\lambda_{2}}{\lambda_{1}+\lambda_{2}}=\frac{e^{2 j \nu}-e^{-2 j \nu} \tan ^{2} \gamma}{e^{2 j \nu}+e^{-2 j \nu} \tan ^{2} \gamma}=B .
$$

It yields:

$$
\tan \alpha_{s}=|B| \text { and } \Phi_{\alpha_{s}}=\arg (B)
$$

\subsection{Con-eigenvalue phase ambiguity}

Due to the con-eigenvalue phase ambiguity, Huynen's parameters need to be reevaluated. To overcome this problem, Touzi has proposed to restrict the tilt angles $\theta_{1}=\theta_{R}+\theta_{E}$ and $\theta_{2}=\theta_{R}-\theta_{E}$ domain definition to the interval $[-\pi / 2, \pi / 2]$ [1] . If the tilts angles $\left(\theta_{1}, \theta_{2}\right)$ are solution of (7), then $\left(\theta_{1} \pm\right.$

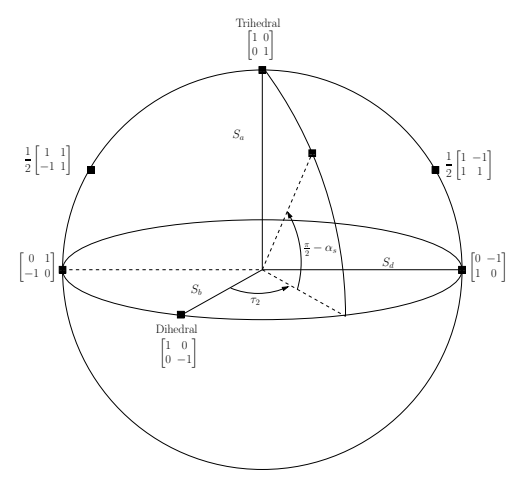

Fig. 1. Symmetric scattering type magnitude $\alpha_{s}$ and helicity $\tau_{2}$ Poincaré sphere $\left(\tau_{2}=0\right.$ and $\left.\Phi_{\alpha_{s}}=0\right)$.

$\left.\pi, \theta_{2} \pm \pi\right),\left(\theta_{1} \pm \pi, \theta_{2}\right)$ and $\left(\theta_{1}, \theta_{2} \pm \pi\right)$ are also solutions of (7). It yields the following three relations:

$$
\begin{aligned}
\mathbf{k}_{\mathbf{P}} & =\mathbf{k}_{\mathbf{P}}\left(\Phi_{s} \pm \pi, \theta_{1} \pm \pi, \theta_{2} \pm \pi, \tau_{1}, \tau_{2}, \mu, \alpha_{s}, \Phi_{\alpha_{s}}\right) \\
& =\mathbf{k}_{\mathbf{P}}\left(\Phi_{s}, \theta_{1} \pm \pi, \theta_{2},-\tau_{1},-\tau_{2}, \mu, \alpha_{s}, \Phi_{\alpha_{s}} \pm \pi\right) \\
& =\mathbf{k}_{\mathbf{P}}\left(\Phi_{s} \pm \pi, \theta_{1}, \theta_{2} \pm \pi,-\tau_{1},-\tau_{2}, \mu, \alpha_{s}, \Phi_{\alpha_{s}} \pm \pi\right) .
\end{aligned}
$$

Those equations are implemented to solve the con-eigenvalue phase ambiguity problem. After this step $\theta_{1}$ and $\theta_{2}$ belong to the interval $[-\pi / 2, \pi / 2]$.

\section{INTERPRETATION}

\subsection{Poincaré Sphere}

To understand the influence of the 4 roll-invariant parameters $\alpha_{s}, \Phi_{\alpha_{s}}, \tau_{1}$ and $\tau_{2}$, the Poincaré sphere representation can be used. Here, only the symmetric scattering type magnitude $\alpha_{s}$ and helicity $\tau_{2}$ Poincaré sphere is shown (Fig. 1). The other spheres can be found in [1]. A symmetric scatterer $\left(\tau_{1}=0\right)$ with a null symmetric scattering type phase $\left(\Phi_{\alpha_{s}}=0\right)$ is uniquely mapped by a point located at a longitude $\tau_{2}$ and a latitude $\pi / 2-\alpha_{s}$ at the surface of this Poincaré sphere.

The symmetric target scattering type phase $\Phi_{\alpha_{s}}$ is the trihedral-dihedral channel phase difference. This roll-invariant parameter can be exploited only under coherence conditions. 
The degree of coherence of $\Phi_{\alpha_{s}}$ (denoted $p_{\Phi_{\alpha_{s}}}$ ) is therefore introduced. Its expression is given by:

$$
p_{\Phi_{\alpha_{s}}}=\frac{\sqrt{\left(\left\langle|a|^{2}-|b|^{2}\right\rangle\right)^{2}+4\left|\left\langle a \cdot b^{*}\right\rangle\right|^{2}}}{\left\langle|a|^{2}+|b|^{2}\right\rangle}
$$

where $a=\cos \alpha_{s} \cos \tau_{1}$ and $b=\sin \alpha_{s} e^{j \Phi_{\alpha_{s}}} \cos \tau_{2}$ for a bistatic polarimetric radar. Therefore, a partially coherent scatterer is represented as a point inside the Poincaré sphere at a distance $p_{\Phi_{\alpha_{s}}}$ from the sphere center.

\subsection{Comparison with the $\alpha / \beta$ bistatic model}

In 2005, S.R. Cloude has proposed to extend the well-known $\alpha / \beta$ model to the bistatic case [8]. The target vector $\mathbf{k}_{\mathbf{P}}$ is defined by means of 8 parameters:

$$
\mathbf{k}_{\mathbf{P}}=\mu e^{j \Phi_{S}}\left[\begin{array}{c}
\cos \alpha \\
\sin \alpha \cos \beta e^{j \delta} \\
\sin \alpha \sin \beta \cos \chi e^{j \gamma} \\
\sin \alpha \sin \beta \sin \chi e^{j \epsilon}
\end{array}\right] .
$$

For the monostatic case $\left(\chi=0\right.$ or $\theta_{R}=\theta_{E}$ and $\tau_{R}=\tau_{E}$ ), the $\alpha$ angle has been widely used to characterize the backscattered mechanism. Indeed, Touzi has proved in [1] that the symmetric scattering type magnitude $\alpha_{s}$ is equal to $\alpha$ for a symmetrical target $\left(\tau_{m}=0\right)$ which corresponds to a wide class of targets including dihedral, trihedral, dipole, ... Fig. 2 shows a comparison between parameters $\alpha$ and $\alpha_{s}$ issued respectively from the $\alpha / \beta$ model and the bistatic TSVM. This plot shows their evolution as a function of the tilt angle $\theta_{2}=$ $\theta_{R}-\theta_{E}$ for different set of target helicity $\tau_{2}=\tau_{R}-\tau_{E}$. Fig. 2(a) and 2(b) are respectively done for $\tau_{1}=0$ and $\tau_{1} \neq 0$. First, it can be seen that $\alpha$ depends on the tilt angle $\theta_{2}$. It yields that, for the bistatic case, $\alpha$ is not a roll-invariant parameter.

In the monostatic case, $\alpha$ and $\alpha_{s}$ are equal for a symmetrical target. This phenomenon is observed in Fig. 2(a) Indeed, a symmetrical target has a null target helicity (i.e. $\tau_{m}=0=$ $\tau_{1} / 2$ ) and the monostatic case is retrieved for $\theta_{R}=\theta_{E}$ (i.e. $\left.\theta_{2}=0\right)$.

It yields that the $\alpha / \beta$ model cannot be directly transposed to the bistatic case to extract a roll-invariant quantity. The bistatic TSVM should be used instead to provide an unique and roll-invariant target decomposition by means of five independent parameters $\alpha_{s}, \Phi_{\alpha_{s}}, \tau_{1}, \tau_{2}$ and $\mu$. As for the monostatic case, those parameters are necessary for an unambiguous description of the backscattering mechanism.

\section{CONCLUSION}

In this paper, a generalization of the Target Scattering Vector Model to the bistatic case has been proposed. Based on the Kennaugh-Huynen decomposition, five parameters, namely

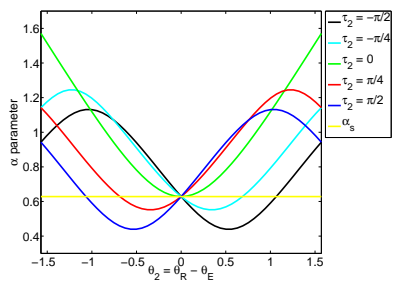

(a)

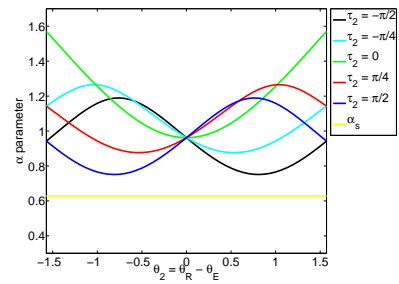

(b)
Fig. 2. Comparison between $\alpha$ and $\alpha_{s}$ as a function of $\theta_{2}$ and $\tau_{2}$ with $\theta_{1}=\pi / 3$ for: (a) $\tau_{1}=0$ and (b) $\tau_{1} \neq 0$

$\alpha_{s}, \Phi_{\alpha_{s}}, \tau_{1}, \tau_{2}$ and $\mu$, are necessary for an unambiguous description of a coherent target. The "monostatic" TSVM has been retrieved as a particular case of the proposed bistatic decomposition. Some comparisons with the so-called $\alpha / \beta$ model parameters have been done. It yields that $\alpha$ is not rollinvariant for the general case of bistatic polarimetry.

Further works will deal with the development of a bistatic incoherent target decomposition in terms of roll-invariant parameters.

\section{Acknowledgment}

The author wish to thank the French Research Agency (ANR) for supporting this work through the EFIDIR project (ANR2007-MCDC0-04, http://www.efidir.fr).

\section{REFERENCES}

[1] R. Touzi, "Target Scattering Decomposition in Terms of RollInvariant Target Parameters," IEEE Transactions on Geoscience and Remote Sensing, vol. 45, no. 1, pp. 73-84, January 2007.

[2] J.R. Huynen, Phenomenological Theory of Radar Targets, Academic Press, 1978.

[3] K. Kennaugh, "Effects of Type of Polarization on Echo Characteristics," Ohio State Univ., Research Foundation Columbus Antenna Lab, Tech. Rep. 389-4, 381-9, 1951.

[4] J.R. Huynen, "Measurement of the Target Scattering Matrix," Proceedings of the IEEE, vol. 53, no. 8, pp. 936-946, August 1965.

[5] A.-L. Germond, Théorie de la Polarimétrie Radar en Bistatique, Ph.D. thesis, Université de Nantes, Nantes, France, 1999.

[6] Z.H. Czyz, "Fundamentals of Bistatic Radar Polarimetry Using the Poincare Sphere Transformations," Technical report, Telecommunications Research Institute, http://airex.tksc.jaxa.jp/pl/dr/20010100106/en, 2001.

[7] C. Titin-Schnaider, "Polarimetric Characterization of Bistatic Coherent Mechanisms," IEEE Transactions on Geoscience and Remote Sensing, vol. 46, no. 5, pp. 1535-1546, May 2008.

[8] S.R. Cloude, "On the Status of Bistatic Polarimetry Theory," in Geoscience and Remote Sensing, IGARSS'05, Seoul, Korea, 2005, pp. 2003-2006. 